\title{
Clinical Assessment of a Mesotherapy Formulation for Skin Rejuvenation in Healthy Volunteers
}

\author{
Anne Grand-Vincent ${ }^{1}$, Sylvie Boisnic ${ }^{2}$, Catherine Salomon ${ }^{3}$, Pascal Prinderre ${ }^{4}$, \\ Philippe Piccerelle ${ }^{4}$ \\ ${ }^{1}$ Aesthetic Medecine Center, Paris, French \\ ${ }^{2}$ Scientific Research and Assessment Group in Dermatology and Cosmetology (GREDECO), Paris, French \\ ${ }^{3}$ Filorga Laboratories, Paris, French \\ ${ }^{4}$ Pharmaceutical Bioengineering Department, Mediterranean Institute of Biodiversity and Marine Ecology, Marseille, French \\ Email: philippe.piccerelle@univ-amu.fr
}

How to cite this paper: Grand-Vincent, A., Boisnic, S., Salomon, C., Prinderre, P. and Piccerelle, P. (2017) Clinical Assessment of a Mesotherapy Formulation for Skin Rejuvenation in Healthy Volunteers. Journal of Cosmetics, Dermatological Sciences and Applications, 7, 291-305.

https://doi.org/10.4236/jcdsa.2017.74026

Received: July 18, 2017

Accepted: November 7, 2017

Published: November 10, 2017

Copyright (ㅇ 2017 by authors and Scientific Research Publishing Inc. This work is licensed under the Creative Commons Attribution International License (CC BY 4.0).

http://creativecommons.org/licenses/by/4.0/

\begin{abstract}
Mesotherapy is a mildly invasive procedure involving several subcutaneous drug injections, which are performed simultaneously. The concept is to inject a very small amount of active compounds (hyaluronic acid, vitamins, antioxidants, enzymes, humectants, ...) and the aim is to stimulate the biosynthetic capacity of fibroblasts, increase collagen and elastin production and to give active essential molecules for a fibroblasts environment. Numerous studies suggest that the mesotherapy procedure improves skin hydration and mechanical properties; however, some results have not been statistically significant. In this study, we investigated an antiaging treatment as a complementary approach to a non-surgical rejuvenating procedure after 5 sessions of mesotherapy. Our main objective was to assess the improvement of face, neck and neckline skin quality, after intradermal microinjections of NCTF $135 \mathrm{HA}^{\star}$. This mixture, manufactured by FILORGA Laboratories, includes a non-cross-linked hyaluronic acid, vitamins, antioxidants, mineral salts and enzymes. A statistically significant decrease in the depth of crow's-feet wrinkles $(43.3 \%)$ and in pore size $(58.5 \%)$ was observed. Also, dermatological scores showed a significant decrease, and data suggest that skin tone was improved with a brighter tone. The injected mixture could then have an action on the skin pigmentation process with a photoprotective effect. Evolution of Dermis density was also investigated and an increase of dermal density and thickness were highlighted, showing an improvement of skin firmness with significant increases of dermis thickness (20\%) and dermis density (24\%), indicating a neocollagen activity.
\end{abstract}




\section{Keywords}

Mesotherapy, Skin Rejuvenation, Multi-Component Solution Crow's-Feet Wrinkles, Pore Size, Dermis Density, Dermis Thickness

\section{Introduction}

Skin aging is a very complex biological and physiological process, divided in two parts: The chronological or intrinsic aging process, associated with the genetic background, and the extrinsic aging, including adverse environmental events such as exposure to solar UV, pollutants, heavy metal, nanoparticles, smoking, stress and lifestyle, which can alter gene expression and skin defects [1]. Skin is exposed daily to extrinsic factors that directly or indirectly cause the generation of free radicals. Oxidative stress plays a key role in skin aging and causes damage on the epidermis and dermis layers [2]. Both the intrinsic and extrinsic aging processes are associated with structural, functional and mechanical skin changes: the loss of tissue, the formation of wrinkles, skin dryness, roughness, yellowness, dyspigmentation and low elasticity [3]. So, one can see histological changes, associated with skin aging, including the accumulation of elastotic material, quantitative changes in collagen, decrease of content and biosynthesis of extracellular matrix proteins (collagen, hyaluronic acid and elastin), decrease of the proliferative capacity of fibroblasts [4]. The structural re-organization of the dermal extracellular matrix highlighted the important role of external factors. In dermatology and cosmetology, antiaging strategies are constantly being developed to overcome nature.

Topical products such as anti-aging cosmetic creams require a prolonged period for few results while plastic surgery involves invasive procedures. Monotherapy, on the other hand, is a mildly invasive procedure involving several subcutaneous drug injections that are performed simultaneously. The concept is to inject a very small amount of active compounds (hyaluronic acid, vitamins, antioxidants, enzymes, humectants, ...) [5] and to increase skin hydration thus improving rejuvenation and skin tone. The aim is to stimulate the biosynthetic capacity of fibroblasts [6], to increase collagen and elastin production and to give active essential molecules for a fibroblasts environment. Various studies suggest that a mesotherapy procedure improves skin hydration and mechanical properties, firmness and viscoelastic properties [7]. However, some results are not statistically significant [8]. This anti-aging treatment is a complementary approach to a non-surgical rejuvenating procedure [6]. A mixed composition and the mechanical needle effects, will influence the final results.

The main objective of this study is to assess the improvement of face, neck and neckline skin quality, after intradermal microinjections of NCTF $135 \mathrm{HA}^{\circ}$. This mixture, manufactured by FILORGA Laboratories, includes a 
non-cross-linked hyaluronic acid, vitamins, antioxidants, mineral salts and enzymes. The study protocol involves 5 sessions of mesotherapy. Biometrological tests were performed by the GREDECO center: dermatological scores, profilometry, standardized photographic assessment, skin color analysis by chromameter, and high frequency ultrasound imaging. The secondary aim is to assess the patient's tolerability.

\section{Subject and Methods}

\subsection{Subject Selection}

Twenty healthy females between 32 and 67 years old (mean age: 49.25 years) were enrolled in the study between January and June 2014. They exhibited a healthy Caucasian skin type on the anatomic area studied. Nine had already received esthetic treatment: wrinkle filling, lifting, abdominoplasty, blepharoplasty and breast enlargement operations.

Two participants stopped after the first cure: the first could not come anymore and the $2^{\text {nd }}$ exhibited urticarial rash at the injection site.

- Inclusion criteria

Age between 35 and 65 years old, skin phototypes I-III patients who consult for wrinkle management, skin hydration and skin tone improvement.

Furthermore, all participants have no adverse effects to mesotherapy, hypersensitivity or allergy to any constituent of the investigated product: NCTF $135 \mathrm{HA}^{\oplus}$

All participants were dedicated to the present study and signed an informed consent form, including not modifying their way of life and no sunbathing.

- Exclusion criteria

Participants with phototype V and over, as well as those enrolled in any clinical evaluation of drugs or medical devices, were excluded.

Also, participants could not be involved in another clinical study and could not use products, techniques or surgery with an anti-wrinkling action.

Furthermore, patients with non-resorbable material fillers, acute inflammation, scars with pigmentation defects, keloid scars, facial herpes, congenital or acquired disorders of the coagulation system, viral or bacterial infection and with a history of auto-immune disease, were excluded. Taking certain medications was another possible cause of the exclusion criteria of the clinical trial, such as oral and injectable corticoid therapy ( $<3$ months), chemotherapy and immunosuppressive therapy ( $<2$ years). Finally, pregnant and lactating women were not included in the clinical trial.

\subsection{Study Design}

The pilot study was set to evaluate the efficacy of NCTF ${ }^{\circledast} 135 \mathrm{HA}$ and consisted of 5 injection sessions at: Day 0, D15, D30, D60 and D90. The injections were given on the face, neck and neckline skin. 
Twenty women were involved: Dr S. Boisnic (GREDEDCO) for evaluation and to Dr A. Grand-Vincent as investigator and trained physician for injection.

The NCTF 135HA has the following composition (Table 1).

Prior inclusion, one visit was performed to check that participants would fit with the inclusion criteria.

During each session, the following volumes were injected:

- $3.36 \mathrm{ml}$ for Session 1;

- $3.72 \mathrm{ml}$ for Session 2;

- $4.47 \mathrm{ml}$ for Session 3;

- $4.92 \mathrm{ml}$ for Session 4 ;

- $5.58 \mathrm{ml}$ for Session 5 .

Data represent the average injected volume.

The first session was conducted without applying a local topical anaesthetic cream. For the following sessions, $60 \%$ of participants applied $\mathrm{EMLA}^{\oplus}$ cream 1 hour before injection.

Evaluations were conducted by Dr Boisnic from GREDECO (Research Group of Evaluation in Dermatology and Cosmetology) and with Dr. Anne Grand-Vincent.

The clinical assessment was implemented by a scoring dermatological system,

Table 1. Composition of $\mathrm{NCTF}^{\circledast} 135 \mathrm{HA}$ (approved CE marking).

\begin{tabular}{cc}
\hline Class & Components \\
\hline Vitamin A (Retinol) \\
Vitamin C (Ascorbic acid) \\
Vitamin B1 (Thiamine) \\
Vitamin B2 (Riboflavin) \\
Vitamin B3 (Nicotinamide) \\
Vitamin B5 (Pantothenic acid) \\
Vitamin B6 (Pyridoxin) \\
Vitamin B8 (Biotin) \\
Vitamin B9 (Folic acid) \\
Vitamin B10 (p-aminobenzoic acid) \\
Vitamin B12 (Cyanocobalamin) \\
Calcium chloride, Potassium chloride, Magnesium \\
sulfate, Sodium acetate, sodium chloride, sodium \\
dihydrogen phosphate \\
Alanine, Asparagine, Arginine, Aspartic acid, \\
Cystine, Glutamine, Glutamic acid, Glycin, \\
Histidine, Hydroxyproline, Isoleucine, Leucine, \\
Lysine, Methionine, Ornithine, Phenylalanine, \\
Proline, Serine, Taurine, Threonine, Tryptophan, \\
Tyrosine, Valine. \\
Amino acids \\
Deoxyadenosine, Deoxycytidine, Deoxyguanosine, \\
Deoxythymidine, Methylcytosine, TPP, CoA, FAD, \\
NADP, UTP, Glutathione, Non-reticulated \\
sodium hyaluronate \\
\\
Antioxidants and other compounds
\end{tabular}


with regard to the depth of wrinkles, on a scale of $0-4$ (4 for deeper wrinkles) and skin radiance (4 for dull skin). The biometrological assessment was carried out using various techniques:

- Standardized photos of face and 3/4 (Canon EOS 50D, Macro-14EX ring) with lamps recreating the daylight without flash (Lastolite ${ }^{\circledR}$ Ray D8 C5600);

- Profilometric (SkinStation ${ }^{\oplus}$ ) measurements of fine lines and crow's feet wrinkles: 3D visualization with color scale to assess wrinkle volume: red for deeper wrinkles, yellow for the skin surface and blue-green for raised skin relief;

- Macrophotography (Proscope $\times$ $\times 30$ ) to assess average pore size in micrometer unit;

- Chromametric (Chromameter Konica Minolta CR/DP-400) analysis to assess skin tone homogeneity L parameter;

- Determination of the dermis thickness and density on the cheek, using High Frequency Ultrasound method (Monaderm ${ }^{\oplus} 20 \mathrm{MHz}$ ).

All results were expressed in the form of average +/- standard deviation, the statistical test was a test of Student.

The method of High Frequency Ultrasound is a non-invasive technique used to evaluate dermal changes with aging [9]. The presence and the degree of sub epidermal low echogenic band (SLEB), is photoaged related. It has been demonstrated that low echogenicity correlates to UV irradiation exposure. The echogenicity is the ability of a tissue to reflect ultrasound echogenic signals and comes from a network of fibers and more particularly collagen and elastin fibers.

- Primary Objective

Evaluation of skin quality for the face, neck and neckline skin.

All in-vitro evaluations were conducted at GREDECO:

- Dermatologic scores;

- Prolilometry;

- Photos;

- Macroscopy;

- Chromametry;

- Cutaneaous Echography (high frequency).

- Secondary Objectives

$>$ Tolerance;

$>$ Satisfaction rating of physician;

$>$ Satisfaction rating of participant.

\section{Discussion}

In our study, a statistically significant decrease of $43.3 \%$ (score of 1.23 versus 2.17; $\left.\mathrm{p}=0.0214 \times 10^{-9}\right)$ in the depth of crow's-feet wrinkles was observed after 5 sessions of mesotherapy. These results were achieved by dermatological scoring (Lemperle scale). This decline had already started after 15 days and increased after 90 days of treatment (Figure 1).

One can see that mesotherapy visibly reduces the volume of crow's-feet wrinkles. The results of profilometric measurements have shown a significant 


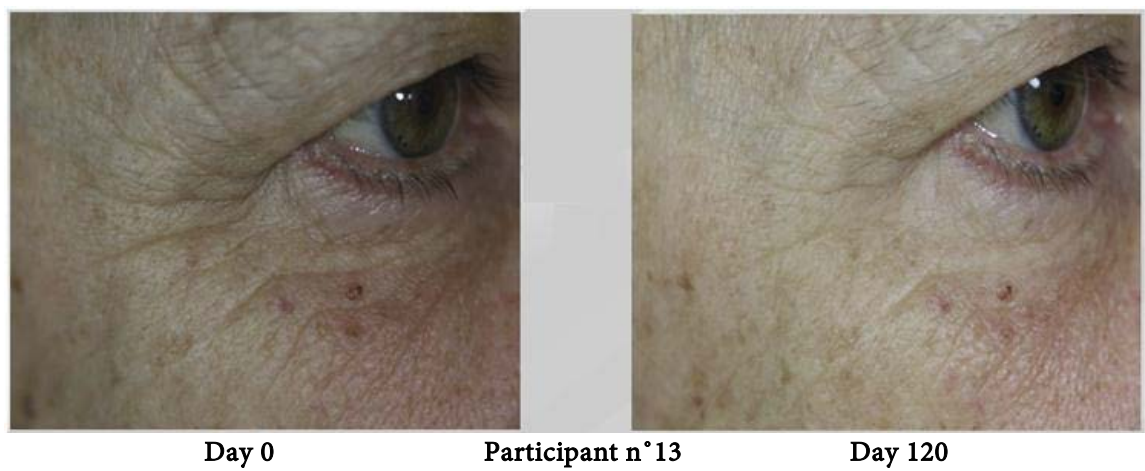

Figure 1. Evolution of crow's-feet wrinkles after treatment on Subject 13.

decrease of wrinkle volume by $32.9 \%$ (mean depth of 18.10 versus $26.98 \mathrm{~V} / \mathrm{mm}^{2}$, $\left.\mathrm{p}=0.0265 \times 10^{-6}\right)$ (Figure 2).

To complete the assessment of mesotherapy on mechanical skin properties, we tried to show treatment efficacy on the reduction of pore size. Our study highlighted a clear decrease in pore size from the $15^{\text {th }}$ day, as shown in Figure 3. After 120 days of treatment, the study found that there was a change in pore size, with a statistically significant decrease of $58.5 \%$, the size decreased from 167.39 $\mu \mathrm{m}$ to $69.44 \mu \mathrm{m}\left(\mathrm{p}=0.0174 \times 10^{-9}\right)$. As indicated in the picture (in the same magnification factor). This parameter is connected with sagging skin.

One can explain the efficiency on skin mechanical properties (increase of the skin firmness pulling a reduction of the size of the pores) by analyzing the mixture composition and the technique (Figure 4).

The NCTF $135 \mathrm{HA}^{\oplus}$ product consists of a mixture of vitamins, inorganic salts, antioxidants, amino acids and hyaluronic acid [9] and is able to maintain cell proliferation and induce enhanced mRNA expression of collagen I, MMP1 and TIMP-I in Human Skin Fibroblast cells for a least 11 days in culture. In a study, Jäger et al. tested two mixtures on fibroblasts culture. It was revealed that a vitaminic compound $\left(\right.$ Soluvit $\left.{ }^{\circledR}\right)$, provides pro-apoptotic process. Hyaluronic acid can induce col-1 production in HSF by interaction through receptors, including CD44: enhancement of cell activity, synthesis of collagen, elastin and HA (9]. Prikhnenko et al. reported results concerning preclinical in vitro data of NCTF effects on the proliferation and synthesis of extracellular matrix and antiradical activity [10].

Moreover, hyaluronic acid plays a key role in the hydration of the extracellular space. HA promotes an enabling environment for cell development and allows inflammatory modulation [11]. Vitamin A regulates the epidermis is turnover and it should be considered as an anti-drying agent. Vitamin B complex is involved in cell metabolism and allows scavenge ROS; Vitamin $\mathrm{C}$ acts as a very important antioxidant and induces collagen synthesis [12]. Vitamin $\mathrm{E}$ is an antioxidant and moisturizer compound [13]. Topical antioxidants can down-regulate free radical-mediated pathways that damage skin [14].

Ascorbic acid is involved in the function of different enzymes and in collagen 


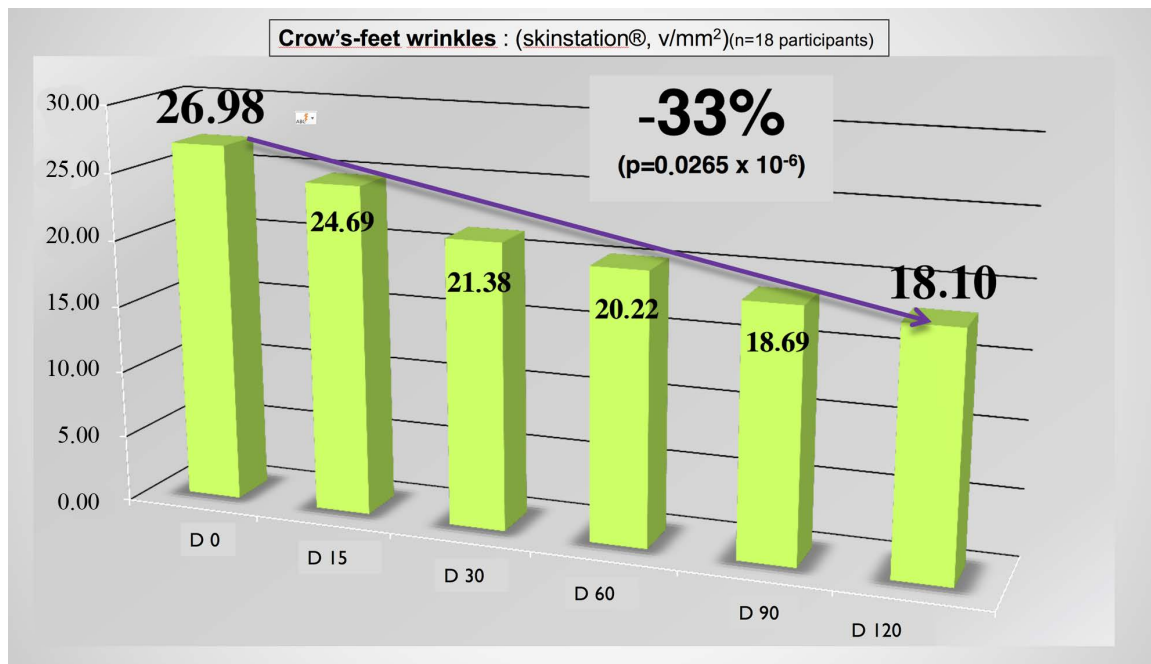

Figure 2. Decrease of crow's-feet mean depth wrinkles $\left(\mathrm{V} / \mathrm{mm}^{2}\right)$ during treatment.

\section{Pore size}

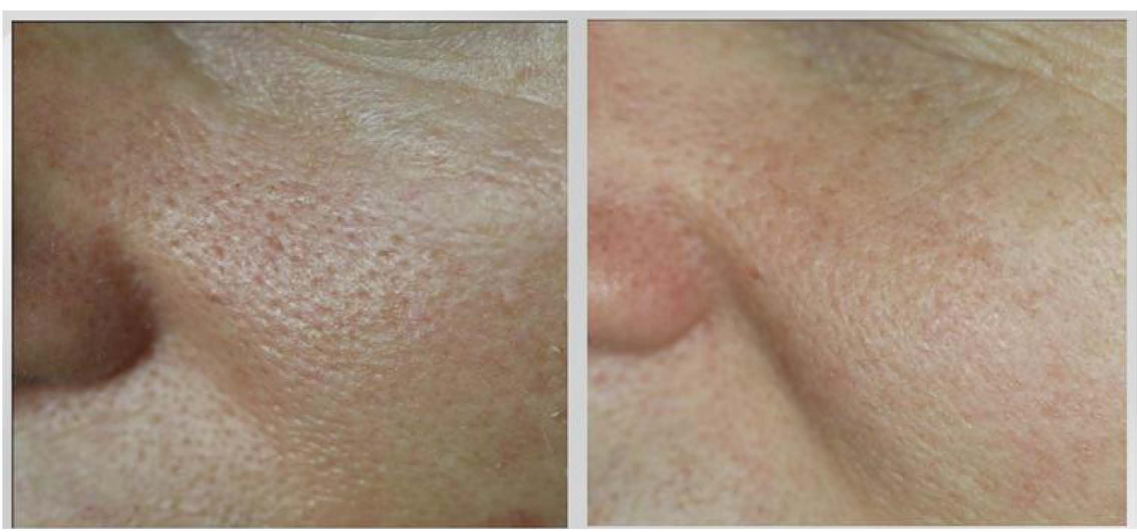

Day 0

Participant $\mathrm{n}^{\circ} 5$

Day 120

Figure 3. Pore size before and after treatment for participant 5.

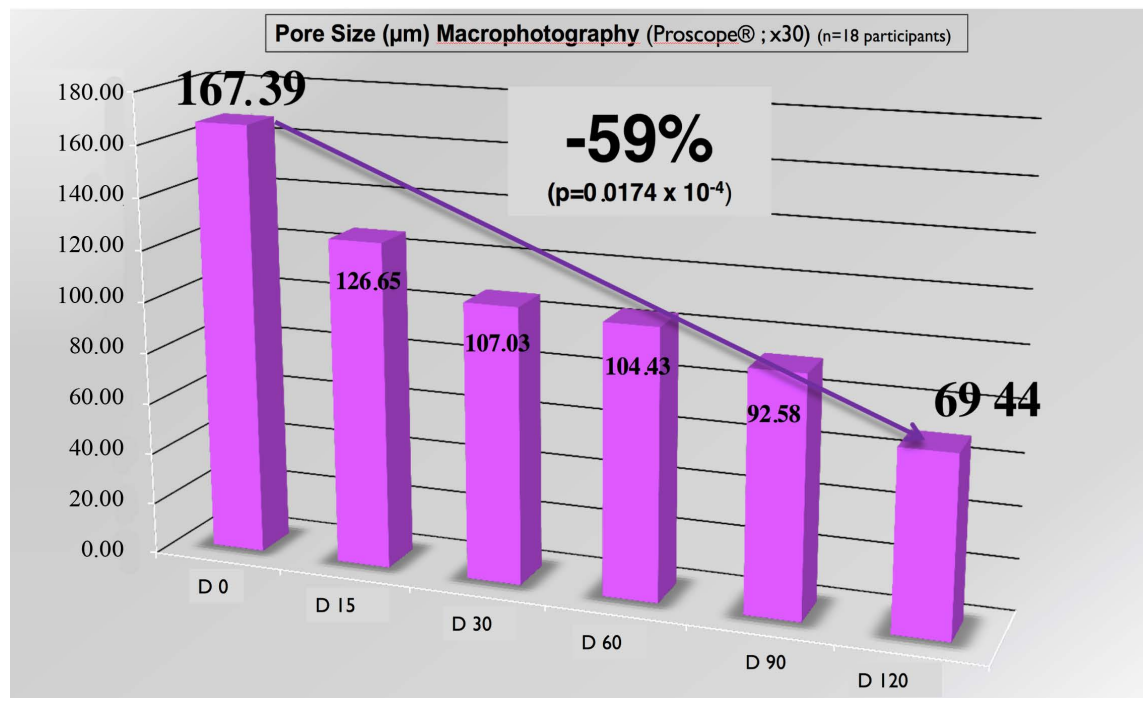

Figure 4. Pore size reduction during treatment. 
synthesis. Vitamin C is a co-factor for hydroxylation of prolyl and lysyl residues of procollagen [15]. It promotes the enzyme activity and enhances the mRNA level of collagen I and III [16].

Loss of proteoglycan hydrophilic properties and glycation products in the papillary dermis, were highlighted by a High Frequency Ultrasound method: The formation of subepidermal low echogenicity band. Physiologically, the Grenz zone is a hypoechoic area with elastotic material. After treatment with topical vitamin C, the ultrasound analysis showed a hyperechoic area [17]. The re-organization of collagen-1 microfibrils explains the action of ascorbic acid Figure 5 [18].

One can see a decrease of more than $50 \%$ at the end of treatment, assuming a significant increase in skin pigmentation homogeneity.

Radiance dermatological scores and the Chromametric biometrological method indicated an improvement of the skin's appearance: Radiance scores showed a significant decrease of score values of $49 \%, 32 \%$ (mean score 1.48 versus 2.94 ; $\mathrm{p}$ $\left.=0.0165 \times 10^{-7}\right)$. These values suggest that skin tone was improved, with a brighter tone. Chromametric measurements showed a statistically significant decrease of the gap of pigmentation between the interior and exterior of the cheek ( $L^{\star}$ parameter). This decrease suggests better skin homogeneity. Our study shows a significant increase of $52.13 \%(1.01$ versus $2.11 ; \mathrm{p}=0.0012)$ of the skin homogeneity after treatment.

Results obtained with chromameter measurements showed an illuminating and brightening activity. The injected mixture was able to have an action on the skin pigmentation process with a photoprotective effect [19]. One can assume that antioxidants, particularly ascorbic acid [18] [20], niacinamide [21] and GSH [22] have skin whitening properties. Niacinamide is a well-known vitamin that decreases melanosome transfer [23] with a very interesting light effect [24]. As for ascorbic acid, it interacts with copper ion at the tyrosinase activity site with strong antioxidant properties [25] and anti-inflammatory effects [20] (Figure 6 and Figure 7).

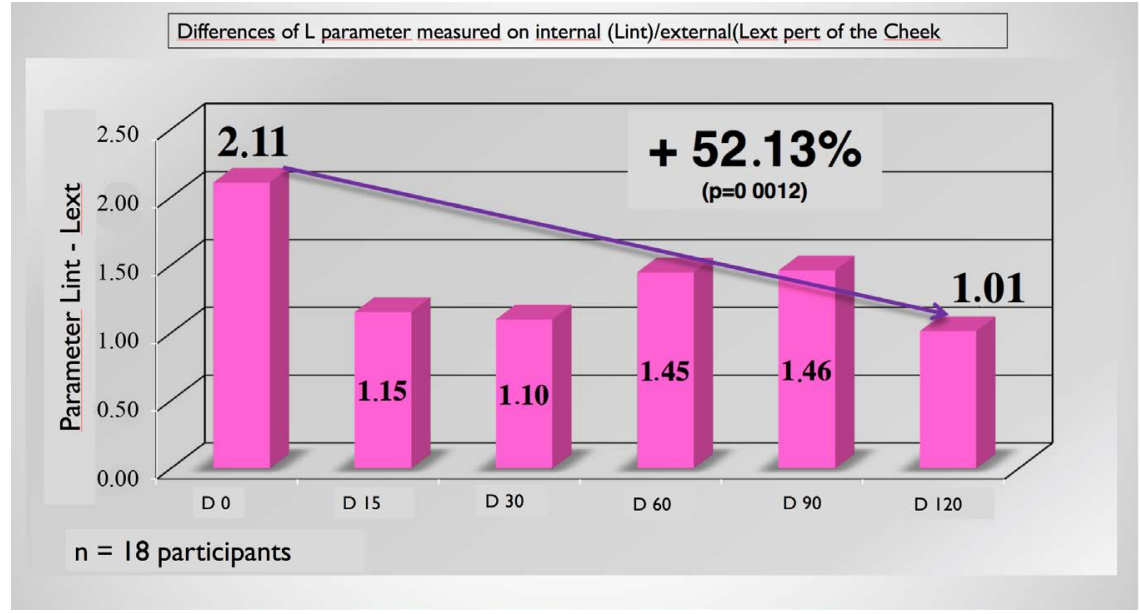

Figure 5. Evolution of L parameter differences with treatment duration. 


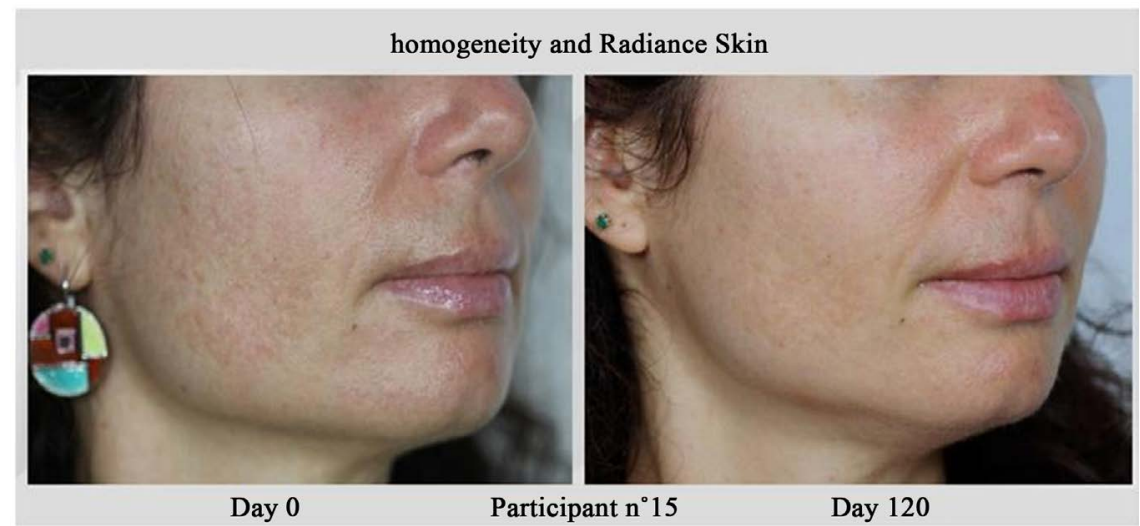

Figure 6. Comparison of homogeneity and skin radiance before and after treatment.

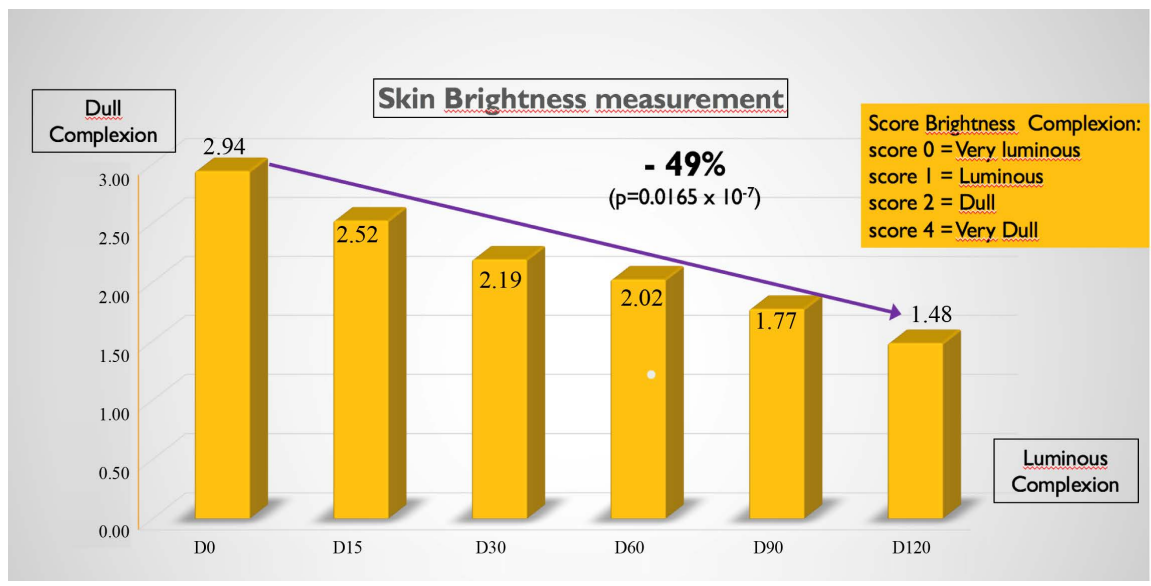

Figure 7. Evolution of Skin Brightnesss core with treatment duration.

Buz et al., in a study concerning the use of GSH in burns, explored the efficiency of mesotherapy in the treatment of dermal burns. The best result of the wound healing process is achieved with glutathione, compared with taurine and L-carnitine treatment. These compounds act as anti-glycan molecules, decreasing the glycation effect of ROS on glycogen fibers. Then, the oxidized thiol form of glutathione is an effective inhibitor of metalloproteinase activity [26]. GSH plays a significant role as a synergistic antioxidant in living tissues by maintaining Vit $\mathrm{C}$ and Vit $\mathrm{E}$ in their reduced forms [27]. GSH is considered as a very effective compound to protect or improve aging skin [28] and the wound healing process [29].

A study of wound healing in aged rats, showed that a topical application of a mix of aminoacids, modulated an inflammatory response, thus increasing endothelial nitric oxide synthase and the Transforming growth factor (TGF-b1) [30]. The activation and proliferation of fibroblasts decrease healing time, allowing the acceleration of cutaneous wound healing in elderly patients [31]. Although arginine supplementation is considered to promote the wound healing process [32].

High Frequency Ultrasound (HFU) is one of the reference techniques used to 
evaluate age related skin changes. One can see in Figure 8 the evolution on low-echogenic band like structure (SLEB) with age [33].

HFU was used to evaluate treatment efficacy by carrying measurement before and after treatment on 2 participants: Figure 4 and Figure 5.

Before the multiple micro-injections of NCTF $135 \mathrm{HA}$ product, one can observe low-echogenic band-like structure (SLEB], which is considered as an indicator of aged skin and more particularly of photo-aged skin [34]. In fact, dermal collagen type, content, structure and interactions with physiological middle of EMC (water, electrolytes), influence dermal echogenicity. The degradation and structure changes of collagen fibers, are responsible for low echogenicity (Figure 9 and Figure 10).

The assessment by High Frequency Ultrasound shows an improvement of skin firmness with an increase of dermis thickness of $20.15 \%$ (1.6 versus 1.34 $\mathrm{mm} ; \mathrm{p}=0.000023)$ and a significant increase of the dermis density of $24.19 \%$ (61.41 versus $49.45 \% ; \mathrm{p}=0.000011)$, indicating a neo-collagenesis activity (Figure 11 and Figure 12).

In our study, we highlighted significant changes in SLEB density related to an

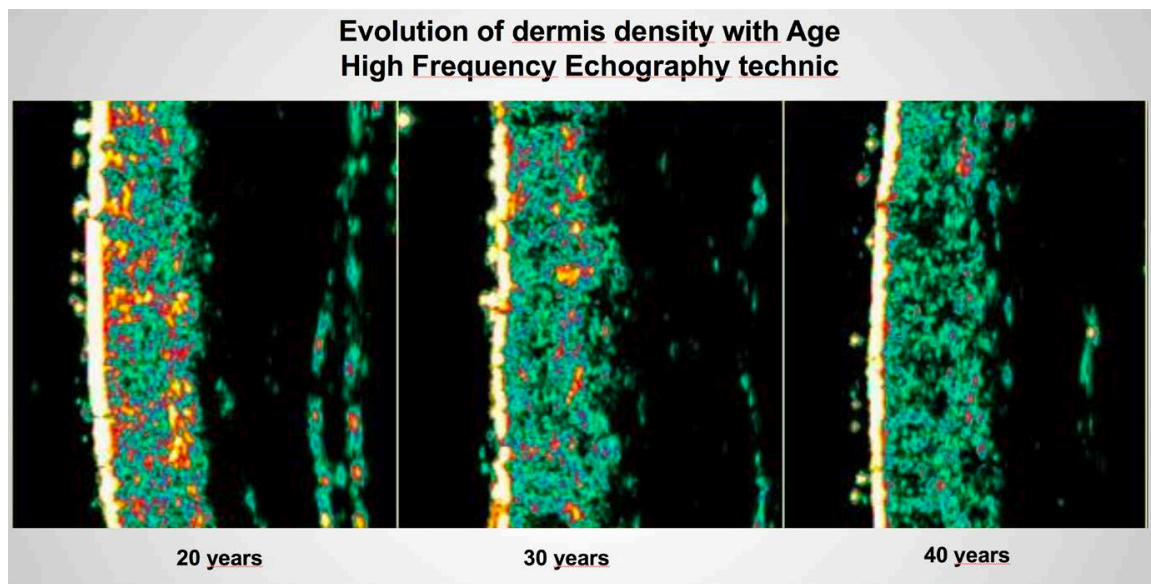

Figure 8. Evolution of Dermis Density with age (Monaderm ${ }^{\circledR}$ website).

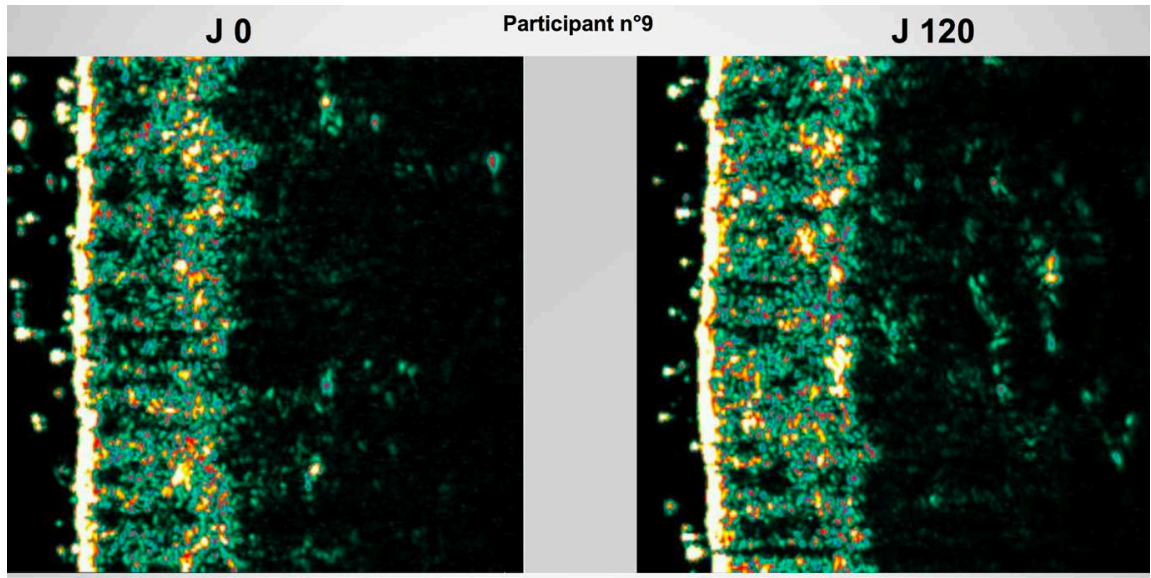

Figure 9. Evolution of Dermis density before/after treatment on participant 9. 


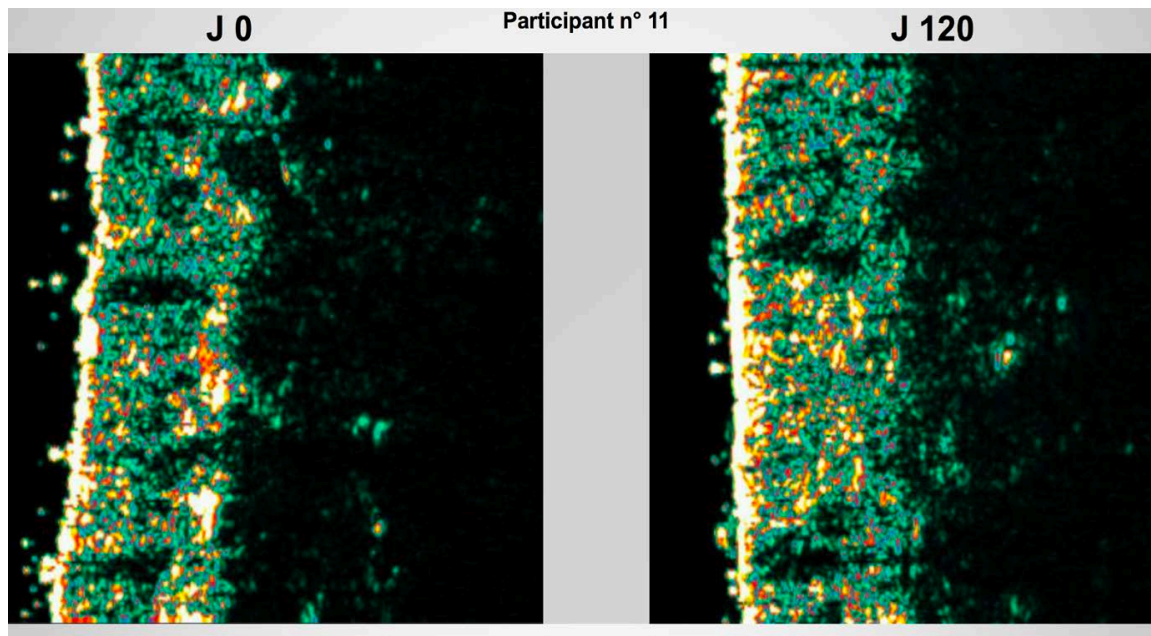

Figure 10. Evolution of Dermis density before/after treatment on participant 11.

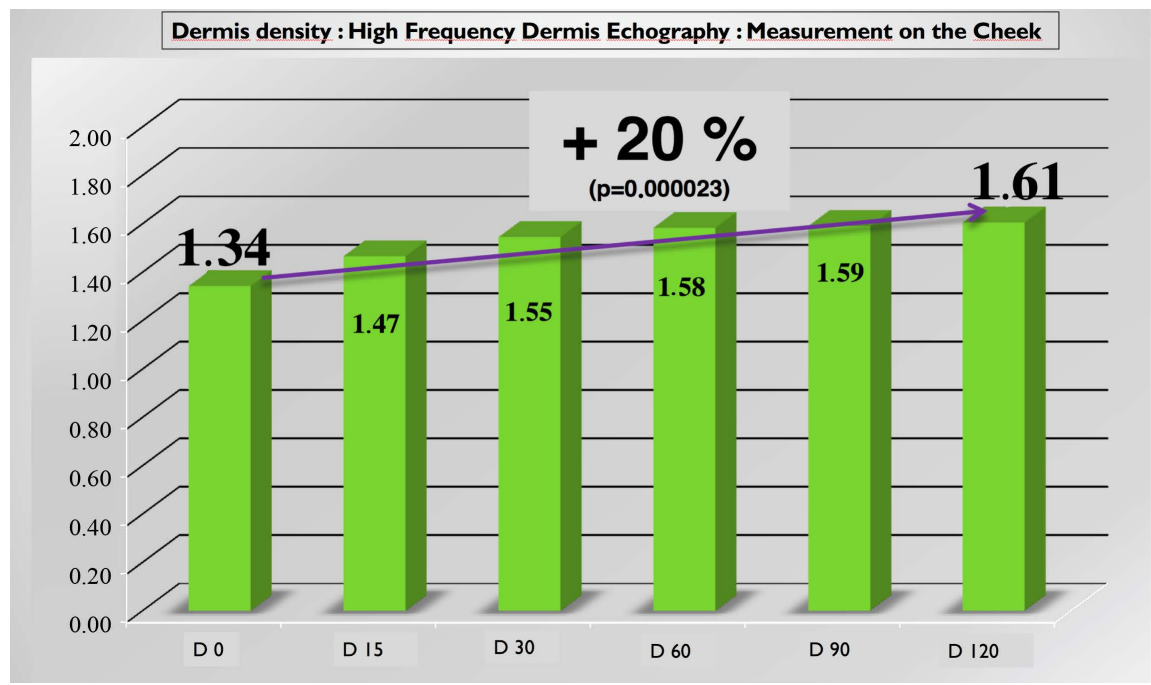

Figure 11. Increases of dermis thickness $(\mathrm{mm})$ during treatment.

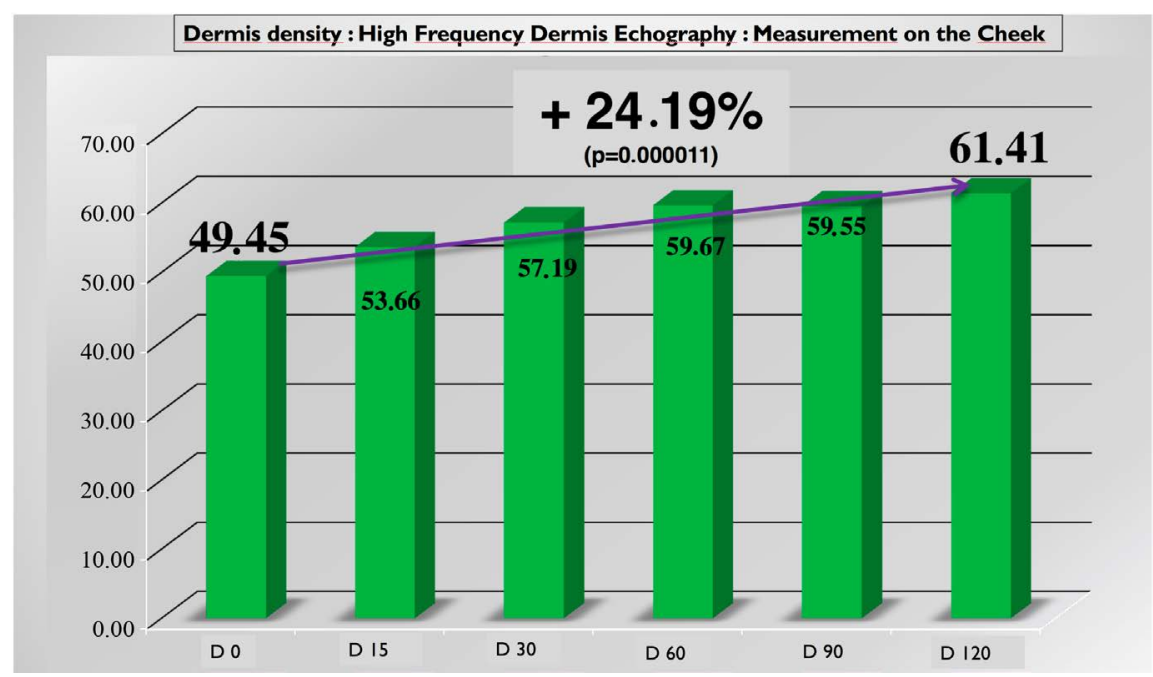

Figure 12. Increases of dermis density (\%) during treatment. 
increase of dermal density and thickness after 120 days of treatment. Skin improvement may be explained by different actions: stimulation of injections and induced low inflammatory process and localized vasodilatation [35]. Injections and active compounds contribute to stimulate elastin and collagen synthesis.

Tedeschi et al. showed that mesotherapic treatment based on Hyaluronic acid product increased the dermal collagen density with fibroblast activation and confirm that mesotherapy may be useful in the treatment of skin aging and photoaging. [36]. Moreover, mesotherapy with HA, improves skin hydration, as well as the viscoelastic properties of the skin. Laccaruba showed that topical HA is a very interesting treatment of human skin atrophy [37]. So, UV irradiation not only decreases the content of HA, but also the expression of CD44 receptors in mice [38] [39]. In 2015, Crisan et al. [17] highlighted that vitamin C improves skin structure and reconstitutes the loss of interstitial collagen by using High Frequency Ultrasound.

Ultrasonic data suggest that collagen synthesis increased significantly, related to the decrease of low echogenic pixels. After treatment, different studies underline the major role of ascorbic acid in the remodeling and the integrity of Extra Cellular Matrix. [40] [41]. Vitamin C prevents the inactivation of enzymes involved in the biosynthesis of collagen: Collagen prolyl 4-hydroxylases [17].

\subsection{Global Satisfaction of the Physician and Patients}

Global satisfaction assessment was done at the end of $5^{\text {th }}$ and last treatment. The physician was satisfied of very satisfied in $100 \%$ of the case, very satisfied in $74 \%$ (14/18 patients). The patients were satisfied or very satisfied in $95 \%$ of the case (17/18 patients), very satisfied in $45 \%$ (8/12 patients).

\subsection{Safety and Tolerance after Injection}

Tolerance after injection was rated as excellent, the local side effects were limited with some cases of erythema, bruises and superficial transient bleeding. One out of twenty participants was discontinued following an allergic reaction at puncture sites. Another subject was excluded for medical problems, not related to the treatment.

\section{Conclusion}

This study demonstrated the efficiency and the tolerance of a multicomponent mesotherapy formulation, improving skin elastic parameters and skin radiance. Mesotherapy technique with a multicomponent solution, can decelerate the signs of ageing and visibly rejuvenate the skin. This improvement is associated with this specific technique and the use of mixed active ingredients, which protect the cells from the damaging action of free-radicals, increasing the collagen synthesis by activation of fibroblasts. Moreover, active ingredients have a very interesting effect on the inflammatory process. The dermic high frequency ultrasound confirmed biometrological tests and dermatological scores, by showing a 
marked improvement in skin conditions.

\section{Comments}

Brief information and informed consent were drawn up for this observational study.

\section{Conflict of Interest}

The study was commissioned by FILORGA Laboratories.

Dr. Catherine Salomon is the medical director of Filorga Laboratories.

\section{References}

[1] Vierkötter, A. and Krutmann, J. (2012) Environmental Influences on Skin Aging and Ethnic-Specific Manifestations. Dermato-Endocrinology, 4, 227-231. https://doi.org/10.4161/derm.19858

[2] Kammeyer, A. and Luiten, R.M. (2015) Oxidation Events and Skin Aging. Ageing Research Reviews, 21, 16-29. https://doi.org/10.1016/j.arr.2015.01.001

[3] Ganceviciene, R., Liakou, A., Theodoridis, A., Makrantonaki, E. and Zouboulis, C. (2012) Skin Anti-Aging Strategies. Dermato-Endocrinology, 4, 308-319.

[4] Makrantonaki, E. and Zouboulis, C. (2007) Molecular Mechanisms of Skin Aging State of the Art. Annals of the New York Academy of Sciences, 1119, 40-50.

[5] Latha, P. and Vandana, K.R. (2011) Mesotherapy-A Review. International Journal of Advanced Pharmaceutics, 1, 19-29.

[6] Iorizzo, M., De Padova, M.P. and Tosti, A. (2008) Biorejuvenation: Theory and Practice. Clinics in Dermatology, 26, 177-181.

[7] Kerscher, M., Bayrhammer, J. and Reuther, T. (2008) Rejuvenating Influence of a Stabilized Hyaluronic Acid-Based Gel of Non Animal Origin on Facial Skin Aging. Dermatologic Surgery, 34, 720-726.

[8] El-Domyati, M., et al. (2012) Efficacy of Mesotherapy in Facial Rejuvenation: A Histological and Immunohistochemical Evaluation. International Journal of Dermatology, 51, 913-919. https://doi.org/10.1111/j.1365-4632.2011.05184.x

[9] Jäger, C., Brenner, C., Habicht, J. and Wallich, R. (2011) Bioactive Reagents Used in Mesotherapy for Skin Rejuvenation in Vivo Induce Diverse Physiological Processes in Human Skin Fibroblasts in vitro-A Pilot Study. Experimental Dermatology, 21, 70-80.

[10] Prikhnenko, S. (2015) Polycomponentmesotherapy Formulations for the Treatment of Skin Aging and Improvement of Skin Quality. Clinical, Cosmetic and Investigational Dermatology, 8, 151-157.

[11] Dessy, L.A., Trignano, E. and Scuderi, N. (2008) Randomized Prospective Study on the Efficacy of a New Revitalizing Filler Composed of Hyaluronic Acid. Giornale Italiano di Dermatologia e Venereologia, 143, 161-165.

[12] Geesin, J.C., et al. (1993) Regulation of Collagen Synthesis in Human Dermal Fibroblasts by the Sodium and Magnesium Salts of Ascorbyl-2-Phosphate. Skin Pharmacology, 6, 65-71. https://doi.org/10.1159/000211089

[13] Lin, J.Y., Selim, M.A., Shea, C.R., et al. (2003) UV Photoprotection by Combination Topical Antioxidants Vitamin C and Vitamin E. Journal of the American Academy of Dermatology, 48, 866-874. https://doi.org/10.1067/mjd.2003.425 
[14] Savoia, A., Landi, S. and Baldi, A. (2013) A New Minimally Invasive Mesotherapy Technique for Facial Rejuvenation. Dermatology and Therapy, 3, 83-93. https://doi.org/10.1007/s13555-012-0018-2

[15] Myllyharju, J. (2003) Prolyl 4-Hydroxylases, the Key Enzymes of Collagen Biosynthesis. Matrix Biology, 22, 15-24.

[16] Nusgens, B.V., Humbert, P., Rougier, A., et al. (2001) Topically Applied Vitamin C Enhances the mRNA Level of Collagens I and III, Their Processing Enzymes and Tissue Inhibitor of Matrix Metalloproteinase 1 in the Human Dermis. Journal of Investigative Dermatology, 116, 853-859. https://doi.org/10.1046/j.0022-202x.2001.01362.x

[17] Crisan, D., Roman, I., Crisan, M. and Badea, R. (2015) The Role of Vitamin C in Pushing Back the Boundaries of Skin Aging: An Ultrasonographic Approach. Clinical, Cosmetic and Investigational Dermatology, 8, 463-470. https://doi.org/10.2147/CCID.S84903

[18] Kameyama, K., Saka, C., Kondoh, S., et al. (1996) Inhibitory Effect of Magnesium L-ascorbyl-2-phosphate (VC-PMG) on Melanogenesis in vitro and in Vivo. Journal of the American Academy of Dermatology, 34, 29-33.

[19] Sparavigna, A., Tenconi, B. and De Ponti, I. (2015) Antiaging, Photoprotective, and Brightening Activity in Biorevitalization: A New Solution for Aging Skin. Clinical, Cosmetic and Investigational Dermatology, 8, 57-65. https://doi.org/10.2147/CCID.S77742

[20] Farris, P.K. (2005) Topical Vitamin C: A Useful Agent for Treating Photoaging and Other Dermatologic Conditions. Dermatologic Surgery, 31, 814-817. https://doi.org/10.1111/j.1524-4725.2005.31725

[21] Hakozaki, T., Takiwaki, H., Miyamoto, K., Sato, Y. and Arase, S. (2006) Ultrasound Enhanced Skin-Lightening Effect of Vitamin C and Niacinamide. Skin Research and Technology, 12, 105-113. https://doi.org/10.1111/j.0909-752X.2006.00186.x

[22] Ebanks, J.P., Wickett, R., Boissy, R. and Raymond, E. (2009) Mechanisms Regulating Skin Pigmentation: The Rise and Fall of Complexion Coloration. International Journal of Molecular Sciences, 10, 4066-4087.

https://doi.org/10.3390/ijms10094066

[23] Hakozaki, T., Minwalla, L., Zhuang, J., Chhoa, M., Matsubara, A., Miyamoto, K., et al. (2002) The Effect of Niacinamide on Reducing Cutaneous Pigmentation and Suppression of Melanosome Transfer. British Journal of Dermatology, 147, 20-31. https://doi.org/10.1046/j.1365-2133.2002.04834.x

[24] Rendona, M. and Horwitz, S. (2012) Topical Treatment of Hyperpigmentation Disorders. Annales de dermatologie et de vénéréologie [Annals of Dermatology and Venereology], 139, S153-S158.

[25] Panich, U., Tangsupa, V., Onkoksoong, T., Kongtaphan, K., Kasetsinsombat, K., Akarasereenont, P., et al. (2011) Inhibition of UVA Mediated Melanogenesis by Ascorbic Acid through Modulation of Antioxidant Defense and Nitric Oxide System. Archives of Pharmacal Research, 34, 811-820.

https://doi.org/10.1007/s12272-011-0515-3

[26] Upadhya, G.A. and Strasberg, S.M. (2000) Glutathione, Lactobionate, and Histidine: Cryptic Inhibitors of Matrix Metalloproteinases Contained in University of Wisconsin and Histidine/Tryptophan/Ketoglutarate Liver Preservation Solutions. $\mathrm{He}$ patology, 31, 1115-1122. https://doi.org/10.1053/he.2000.6780

[27] Deveci, M., Öztürk, S., Bayram, Y., Aydın, A., Eken, A. and Sengezer, M. (2005) Topical Application of Glutathione in the Treatment of Diabetic Wounds. Turkish 
Journal of Plastic Surgery, 13, 179-184.

[28] Watanabe, F., Hashizume, E., Chan, G.P. and Kamimura, A. (2014) Skin-Whitening and Skin-Condition-Improving Effects of Topical Oxidized Glutathione: A Double-Blind and Placebo-Controlled Clinical Trial in Healthy Women. Clinical, Cosmetic and Investigational Dermatology, 7, 267-274. https://doi.org/10.2147/CCID.S68424

[29] Kopal, C., Deveci, M., Ozturk, S. and Sengezer, M. (2007) Effects of Topical Glutathione Treatment in Rat Ischemic Wound Model. Annals of Plastic Surgery, 58, 449-455. https://doi.org/10.1097/01.sap.0000241681.57965.35

[30] Wolfs, B., et al. (2009) Role of Arginine in Superficial Wound Healing in Man. Nitric Oxide, 21, 175-183.

[31] Corsettia, G., D’Antonab, G., Saverio, G. and Rezzania, R. (2010) Topical Application of Dressing with Aminoacids Improves Cutaneous Wound Healing in Aged Rats. Acta Histochemica, 112, 497-507.

[32] Debats, I.B., Wolfs, T.G., Gotoh, T., Cleutjens, J.P., Peutz-Kootstra, C.J. and Van der Hulst, R.R. (2009) Role of Arginine in Superficial Wound Healing in Man. Nitric Oxide, 21, 175-183.

[33] Gniadecka, M. (2001) Effects of Ageing on Dermal Echogenicity. Skin Research and Technology, 7, 204-207. https://doi.org/10.1034/j.1600-0846.2001.70310.x

[34] Sandby-Moller, J. and Wulf, H.C. (2004) Ultrasonographic Subepidermal Low-Echogenic Band, Dependence of Age and Body Site. Skin Research and Technology, 10, 57-63. https://doi.org/10.1111/j.1600-0846.2004.00056.x

[35] Baspeyras, M., et al. (2013) Clinical and Biometrological Efficacy of a Hyaluronic Acid-Based Mesotherapy Product: A Randomised Controlled Study. Archives of Dermatological Research, 305, 673-682. https://doi.org/10.1007/s00403-013-1360-7

[36] Tedeschi, A., Lacarrubba, F. and Micali, F. (2015) Mesotherapy with an Intradermal Hyaluronic Acid Formulation for Skin Rejuvenation: An Intrapatient, Placebo-Controlled, Long-Term Trial using High-Frequency Ultrasound. Aesthetic Plastic Surgery, 39, 129-133. https://doi.org/10.1007/s00266-014-0432-1

[37] Lacarrubba, F., Nardone, B., Tedeschi, A., Nordstrom, R. and Micali, G. (2007) Ultrasound Evaluation of Mesotherapy for Skin Rejuvenation. In: Tosti, A. and De Padova, M.P., Eds., Atlas of Mesotherapy in Skin Rejuvenation, Informa Healthcare Ltd., London.

[38] Lacarrubba, F., Tedeschi, A., Nardone, B. and Micali, G. (2008) Mesotherapy for Skin Rejuvenation: Assessment of the Subepidermal Low-Echogenic Band by Ultrasound Evaluation with Crosssectional B-Mode Scanning. Dermatology and Therapy, 21, S1-S5. https://doi.org/10.1111/j.1529-8019.2008.00234.x

[39] Calikoglu, E., Sorg, O., Tran, C., Grand, D., Carraux, P., Saurat, J.H. and Kaya, G. (2006) UVA and UVB Decrease the Expression of CD44 and Hyaluronate in Mouse Epidermis, Which Is Counteracted by Topical Retinoids. Photochemistry and Photobiology, 82, 1342-1347. https://doi.org/10.1562/2006-02-10-RA-801

[40] Sauermann, K., Jaspers, S., Koop, U., et al. (2004) Topically Applied Vitamin C Increases the Density of Dermal Papillae in Aged Human Skin. BMC Dermatology, 4, 13. https://doi.org/10.1186/1471-5945-4-13

[41] Fisher, G.J., Kang, S., Varani, J., et al. (2002) Mechanisms of Photoaging and Chronological Skin Aging. Archives of Dermatology, 138, 1462-1470. https://doi.org/10.1001/archderm.138.11.1462 